\title{
«Familienaufstellung in der Verhaltenstherapie - Erweiterung des Repertoires oder Modeerscheinung?»
}

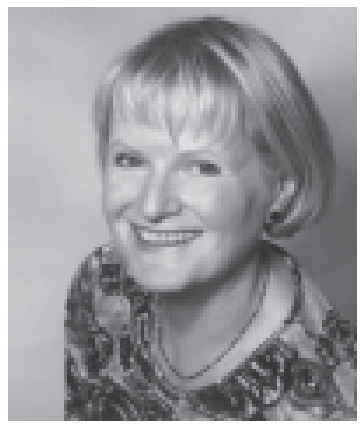

Dr. Maren Langlotz-Weis machte nach dem Studium der Psychologie eine Verhaltenstherapieausbildung am Max-Planck-Institut für Psychiatrie, München, wo sie auch promovierte. Mehrere Jahre lang war sie leitende Psychologin an der Psychosomatischen Fachklinik Bad Dürkheim. Sie machte Weiterbildungen in Psychodrama, Körperpsychotherapie, systemischer und Hypnotherapie. Seit 1985 hat sie eine eigene Praxis als Verhaltenstherapeutin für Erwachsene. Ihr Behandlungsschwerpunkt sind Essstörungen. Daneben ist sie Dozentin, Supervisorin und Vorstandsmitglied am Ausbildungsinstitut IFKV Bad Dürkheim. Das Interview mit ihr führte Prof. Dr. Manfred M. Fichter, Medizinisch-Psychosomatische Klinik Roseneck, Prien, und Psychiatrische Universitätsklinik München (LMU).

Frau Dr. Langlotz-Weis, Sie sind seit vielen Jahren als niedergelassene Psychotherapeutin tätig und haben sich in den letzten Jahren viel mit dem Thema Familienaufstellung befasst. Was versteht man unter Familienaufstellung in einer verhaltenstherapeutischen Gruppentherapie?

Maren Langlotz-Weis: Wenn ein Patient an einer Fragestellung arbeiten will, die direkt oder indirekt mit der Herkunftsoder Jetzt-Familie zusammenhängt, kann die Familienrekonstruktion hilfreich oder weiterführend sein. Dabei geht man davon aus, dass viele irrationale und dysfunktionale Überzeugungen und Interaktionsmuster unter bestimmten sozialen, emotionalen und kognitiven Bedingungen gelernt wurden, z.B. mangelndes Selbstvertrauen auf dem Hintergrund eines strafend-abwertenden Erziehungsstils. Für eine Veränderung bzw. Beeinflussung gilt es, diese Bedingungen noch einmal in ihren verschiedenen Qualitäten zu erleben, Grawe nennt das «Problemaktivierung», um dann neue Bewältigungsversuche aufzubauen. Optimal ist der Gruppenkontext, in dem andere Gruppenmitglieder Familienrollen übernehmen; im Einzelsetting ist auch die Arbeit mit leeren Stühlen oder einem Figurenbrett denkbar.

Einige, die mit Familienaufstellungen in Gruppen arbeiten sagen, dass dies ein besonders wirkungsvolles oder «mächtiges» Verfahren sei. Was sind Ihre Erfahrungen?
Maren Langlotz-Weis: Diese Erfahrungen kann ich nur bestätigen. Eine «Problemaktivierung» wird damit sehr effizient umgesetzt. Oft werden emotional berührende Prozesse angestoßen, z.B. Trauer oder Verzweiflung, die damit aber auch Veränderungsprozesse im Sinne des ganzheitlichen Lernens anstoßen können. Aber auch im Hinblick auf Ressourcenaktivierung und Problemlösefertigkeiten bieten Familienaufstellungen neue Möglichkeiten. Bisher nicht wahrgenommene oder anerkannte Familienmitglieder erweisen sich im Rückblick oft als hilfreich. Lösungen können aufgrund der anschaulichen Vorgehensweise leichter erarbeitet werden.

Sie haben mehrere Jahre in der strikt verhaltenstherapeutischen Abteilung von Prof. Hans Brengelmann am MPI für Psychiatrie gearbeitet, aber auch über den Tellerrand der Verhaltenstherapie gesehen, z.B. mit Ihrer Psychodrama-Ausbildung. Können Sie das theoretische Konzept der Familienaufstellung als Technik in einem verhaltenstherapeutischen Vorgehen kurz skizzieren?

Maren Langlotz-Weis: Mein spezielles Vorgehen ist sehr von meiner psychodramatischen Zusatzausbildung geprägt. Hier unterscheide ich mich von Hellinger, aber auch von manchen systemisch geprägten «Aufstellern». Ein wesentlicher Unterschied besteht im so genannten «Eindoppeln» der Familienmitglieder: Der Patient wählt aus der Gruppe die Stellvertre-

\begin{tabular}{ll}
\hline KARGER & @ 2003 S. Karger GmbH, Freiburg \\
$\begin{array}{l}\text { Fax +49 76145207 14 } \\
\begin{array}{l}\text { E-mail Information@Karger.de } \\
\text { www.karger.com }\end{array}\end{array}$ & $\begin{array}{l}\text { Accessible online at: } \\
\text { www.karger.com/ver }\end{array}$
\end{tabular}

Dr. Maren Langlotz-Weis 
ter aus (auch das ist übrigens ein Unterschied zu den manchmal vom Leiter willkürlich ausgewählten Teilnehmern) und schlüpft kurz in die jeweilige Rolle, unterstützt vom Therapeuten, der mit bestimmten Fragen hilft, dass sich die Stellvertreter in das jeweilige Familienmitglied hineinversetzen können. Hier ergibt sich oft schon ein erster therapeutischer Effekt: Über den Rollentausch und den damit vollzogenen Perspektivenwechsel kann der Patient zu neuen Erkenntnissen oder Einsichten gelangen. Am Ende des Eindoppelns sollte ein Du-Satz formuliert werden können, der die Beziehung zum Patienten repräsentiert und verdichtet; der Spieler merkt sich diesen Satz. Am Ende hört dann der Patient die gesammelten Beziehungsbotschaften aus den jeweiligen Positionen der Familienmitglieder. So gesehen, könnte man die Rekonstruktion der Familie als Veranschaulichung vermuteter, intern gespeicherter Kognitionen und Emotionen der einzelnen Familienmitglieder zum Patienten bezeichnen. An dieser Stelle könnte man die Aufstellung bereits beenden im Sinne einer «Diagnose», mit der auch kognitiv-emotional gut weitergearbeitet werden kann. Geht es mehr um Lösungen oder kognitive Umstrukturierungen, kann von hier aus kreativ, manchmal auch in Richtung Verhaltensaufbau, weitergearbeitet werden, zum Beispiel: «Was ich meinem Vater damals gern gesagt hätte» oder «Ich hätte meiner Mutter gerne noch gedankt, bevor sie starb».

Sie erwähnten im Zusammenhang mit Familienaufstellungen das Stichwort des ganzheitlichen Lernens. Was hat man als Verhaltenstherapeut darunter zu verstehen?

Maren Langlotz-Weis: Ganzheitliches Lernen bezieht sich auf die vier Ebenen der Verhaltensanalyse, nämlich dass Inhalte umso eher wieder erinnert und damit einer Veränderung zugänglich gemacht werden können, je mehr Kodierungsebenen beim Lernen und Neulernen beteiligt sind, also über die Kognition hinaus eben auch die Motorik, das Körpergefühl bzw. die Physiologie und die Emotionen. Die Rekonstruktionsarbeit bietet hier, wie auch sonst das Rollenspiel, viele Anwendungsmöglichkeiten. In der neueren Forschung über die Neurobiologie der Informationsverarbeitung gibt es viele interessante Hinweise und Belege für dieses Konstrukt, z.B. im Hinblick auf das so genannte implizite Gedächtnis oder die Arbeit mit somatischen Markern.

Dient die Familienaufstellung vorrangig der Familiendiagnostik oder der Therapie?

Maren Langlotz-Weis: Eine gute Diagnostik ist manchmal schon die halbe Therapie. Problematiken, Erziehungsstile etc. werden im Sinne einer Diagnostik anschaulich und lösungsorientiert aufbereitet. Darüber hinaus können nach der Aufstellung weitere Schritte erfolgen, z.B. die Frage nach der Wunschfamilie («Wie hätte es sein sollen?»), Probleme des Vergebens oder Perspektivenwechsel: «Was hätte diese Fami- lie, dieses Kind damals gebraucht?» Der Kreativität sind dabei keine Grenzen gesetzt. Der Therapeut kann sein ganzes klinisches Interventionswissen einsetzen, so lange er hypothesengeleitet und lösungsorientiert vorgeht.

Gibt es empirisch fundierte, wissenschaftliche Studien zur Wirksamkeit von verhaltenstherapeutisch ausgerichteter Familienaufstellung?

Maren Langlotz-Weis: Meines Wissens nicht.

Hier gibt es also für Praktiker und Psychotherapieforscher noch viel zu tun, bevor das Verfahren richtig Eingang in die von gesetzlichen Kassen bezahlte Verhaltenstherapie finden kann. Warum sind gerade Familienaufstellungen für Sie so bedeutsam geworden?

Maren Langlotz-Weis: Ich arbeite seit vielen Jahren mit essgestörten jungen Patientinnen, deren häufige familiäre Verstrickung ja bekannt ist. Dort und in der Selbsterfahrung mit angehenden Therapeuten habe ich die Familienaufstellung als ein Verfahren schätzen gelernt, das sehr verdichtet, handlungsorientiert und anschaulich Umlernprozesse fördern kann. Ich rate aber davon ab, Patienten die Ergebnisse der Aufstellung mit den realen Familienmitgliedern diskutieren bzw. manchmal auch aufrechnen zu lassen. Aus meiner Sicht geht es immer um die subjektive innere Repräsentanz der familiären Beziehungen und damit um den Umgang mit sich selbst; eine objektive Wirklichkeit lässt sich nicht rekonstruieren. Trotzdem kann sich die Beziehung zu den jeweiligen Familienmitgliedern verändern, differenzieren oder sogar verbessern.

\section{Welche Vorsichtsmaßnahmen sind bei Familienaufstellungen zu} beachten?

Maren Langlotz-Weis: Da die Aufstellungsarbeit oft emotional aufwühlend ist, sollte sie gut in den therapeutischen Prozess eingebettet sein, das heißt eine vertrauensvolle Heranführung, Beziehungsgestaltung sowie ausreichend Gelegenheit zur Nachbesprechung und Beobachtung langfristiger Auswirkungen sollten gegeben sein. In der Gruppentherapie sollte der Rahmen für eine von der Gruppe getragene Einzelarbeit sichergestellt sein. Der Patient sollte sich sowohl während der Arbeit als auch bei der Auswertung von den anderen Gruppenmitgliedern getragen und respektiert fühlen. Der Patient sollte auch - zumindest in Maßen - emotional belastbar sein. Bei schwer depressiven oder akut psychotischen Patienten sollte man mit Familienaufstellungen zurückhaltend sein.

Bekannt wurden Familienaufstellungen durch Massenveranstaltungen von Bert Hellinger, einem Außenseiter der Psychotherapie auf dem grauen Markt. Hellinger ist eine umstrittene Figur (vergleiche der Spiegel 7/2001 S. 200-202). Was ist es, was diesen früheren Missionar so umstritten macht? 
Maren Langlotz-Weis: Hier könnte man vieles aufzählen, mittlerweile findet die Gegnerschaft Hellingers auch in Veröffentlichungen ihren Niederschlag, z.B. in Colin Goldners «Der Wille zum Schicksal, die Heilslehre des Bert Hellinger». Ich selber habe ihn nicht persönlich erlebt, habe mich aber mit seinen Veröffentlichungen auseinander gesetzt und bemängele den Frauen abwertenden Unterton, z.B. beim Umgang mit Missbrauchsopfern oder Brustkrebspatientinnen, seinen autokratischen Leiterstil und die Überzeugung, zu wissen, was gut für den jeweiligen Protagonisten ist. Schon ehe Hellinger mit Familienaufstellungen begann, gab es diese Methode. Er hat sie nicht erfunden. Er hat aber sehr viel zu ihrer Verbreitung beigetragen. Er hat viele Anstöße gegeben und sich nicht gescheut, sich zu exponieren. Wir sollten uns eher fragen, was so viele Menschen veranlasst, das Heil (wieder) bei einer starken Führerpersönlichkeit zu suchen.

Was halten Sie von seinen apodiktischen Rückmeldungen an Klienten und von seiner besonderen Vorliebe für das Thema «Nationalsozialismus»?

Maren Langlotz-Weis: Hellinger hindert die Patienten, selber Lösungen zu finden. Er sagt ihnen, was gut ist. Fast scheint es, als hole ihn seine Vergangenheit als Priester hier wieder ein. Oft wirken seine Sätze oder Forderungen wie Segnungen oder Flüche im alttestamentarischen Sinne. Darüber hinaus spiegeln seine Rückmeldungen längst überwunden geglaubte, wertkonservative Muster und Überzeugungen, im Sinne einer patriarchalisch-nordeuropäischen Grundordnung wieder. So gibt es auch Kritiker, die ihm latenten Rassismus, Judenfeindlichkeit und ideologische Nähe zum braunen Gedankengut nachsagen; z.B. Fritz Glunk oder Klaus Weber in dem erwähnten Buch.

Was machen Sie bei einer verhaltenstherapeutisch ausgerichteten Familienaufstellung anders als Hellinger und seine Schüler?

Maren Langlotz-Weis: Der wesentliche Unterschied ist, dass die Lösungen mit dem Patienten zusammen erarbeitet werden. Der Therapeut geht mit dem Prozess und weiß nicht von vornherein, was gut für den Patienten ist. Ideen oder Erkenntnisse des Therapeuten werden in Form von Vorschlägen oder Ermutigungen zu Verhaltensexperimenten vorgebracht und im ständigen Rückmelde- und Beobachtungsprozess modifiziert. Die Aufstellung ist eingebunden in einen längeren therapeutischen Kontext, in dem ihre Auswirkungen bearbeitet werden können. Natürlich ist es eine Illusion, völlig wertfrei und ohne Ideologien Lösungen mit dem Patienten erarbeiten zu können; man sollte seine Einstellungen aber transparent machen können, sich ihrer weitestgehend bewusst sein und sie reflektieren können. Deswegen ist eine fundierte Selbsterfahrung in unserem Beruf ja so wichtig.
Halten Sie es für wichtig, nach der unmittelbaren Familienaufstellung eine bestimmte Reihenfolge der Rückmeldungen durch die passiv Zuhörenden, Mitspieler etc. einzuhalten?

Maren Langlotz-Weis: Die Reihenfolge der Aufarbeitung nach der Rekonstruktion ist ebenfalls dem klassischen psychodramatischen Arbeiten entlehnt. Ehe die Spieler Rückmeldung aus den Rollen geben, teilen erst einmal alle Gruppenmitglieder dem Protagonisten im so genannten Sharing mit, ob und wann sie emotional beteiligt waren, was sie unter Umständen aus ihrer eigenen Lebensgeschichte kennen und wo sie sich gut einfühlen konnten. Dadurch wird der Protagonist, der ja viel von sich gezeigt hat und dadurch exponiert war, dass er im Mittelpunkt des Geschehens stand, wieder zurück in die Gruppe geholt und normales Gruppenmitglied. Erst danach sollten die Rollenträger ihre Rückmeldungen geben, und ganz am Schluss ist noch Raum für allgemeine Beobachtungen, Kommentare oder Rückmeldungen. Voraussetzung dafür ist, dass der Patient die Rückmeldungen aufnehmen kann. Unter Umständen ist auch eine kleine Pause sinnvoll.

Bei Hellinger und Nachfolgern spielt «Schuld» eine große Rolle. Wie gehen Sie - in Abgrenzung zu Hellinger - bei Ihren Familienaufstellungen mit dem Thema Schuld um?

Maren Langlotz-Weis: Auch hier gibt es keine Patentlösungen. Das Thema Schuld muss differenziert betrachtet werden. Wenn eine Familienkonstellation z.B. ergibt, dass eine Patienten, die aus einem Seitensprung der Mutter hervorgegangen ist, von dieser so behandelt wird, als wäre sie schuld, dann kann es im Sinne des ganzheitlichen Lernens hilfreich sein, die Patientin zu fragen, wie schwer diese Schuld, die nicht die ihre ist, wiegt; ganz konkret, zum Beispiel mithilfe von aufgeschichteten Büchern in einem Korb oder, phantasievoller, mit diversen Findlingen, und diese Schuld dann der Mutter zurückzugeben. Auch körperlich wird hier deutlich, welche Last bzw. Verantwortung wo hingehört. Gleichzeitig erscheint es wichtig, über Verzeihen oder Vergeben zu arbeiten, wenn die primären Affekte wie Wut, Trauer oder Verzweiflung durchlaufen sind. Ein anderer Aspekt von Schuld bezieht sich auf den Anteil von Verantwortung, den jemand möglicherweise tatsächlich hat, bisher aber nicht sehen wollte, sondern stattdessen «lieber» Schuldgefühle hat. Hier ginge es eher um die Übernahme von Verantwortung für ein Verhalten mit allen dazugehörigen Konsequenzen. Auch für diese Form der Schuldproblematik wäre eine Familienaufstellung mit diversen Lösungsentwürfen denkbar.

Wie ist es zu erklären, dass die Mitspieler in einer Familienaufstellung - nicht nur der Protagonist, sondern auch die anderen - bei und nach Aufstellungen so sicher sind, dass genau diese Ausrichtung, genau dieser Abstand stimmt? 
Maren Langlotz-Weis: Die Systemiker nennen die aufgestellte Familie mit allen Teilnehmern das «wissende Feld». Ob es sich hier um minimale nonverbale Hinweisreize handelt, die dem objektivierbaren Blick nicht zugänglich sind, oder um das von Moreno schon postulierte «Tele», das heißt die Fähigkeit, über Positionierung und Haltung viel über eine fremde Person zu erfahren, oder ob wir ein gemeinsames Wissen über Familienstrukturen haben à la Jung, oder alles zusammen, bleibt wohl noch einige Zeit ein Geheimnis. Im Rahmen verhaltenstherapeutischen Arbeitens ist es jedoch hilfreich, dieses Wissen als Unterstützung und hypothesengenerierende Information zu nutzen, ohne es zur objektivierbaren Wahrheit werden zu lassen.

Familienaufstellungen sind en vogue. Wie Sie schon erwähnten, gibt es bisher keine wissenschaftlichen Untersuchungen zu ihrer Wirksamkeit und ihren Nebenwirkungen. Einige bieten sie marktschreierisch in Großveranstaltungen an und andere experimentieren - wie Sie - damit als Technik im Rahmen einer Verhaltenstherapie bzw. Psychodrama. Welche Perspektiven für Familienaufstellungen in Wissenschaft und Praxis sehen Sie?

Maren Langlotz-Weis: Ich würde mir wünschen, dass wir uns im Sinne Grawes mehr auf eine so genannte Allgemeine Psychotherapie hin entwickeln, in der die wissenschaftlichen Erkenntnisse der gesamten Psychologie und Neurowissenschaften, nicht nur der Lerntheorien, therapeutisch genutzt werden. Vielleicht werden wir damit eines Tages auch bisher exotisch anmutende Verfahren guten Gewissens in unsere therapeutische Praxis einordnen können. Dazu zählen für mich auch die Familienaufstellungen, die sich nach Maßgabe klinisch erfahrener Therapeuten bereits als hilfreich und effektiv erwiesen haben; ihren statistisch signifikanten Wirksamkeitsgrad könnten sie in einer umfassend konzipierten anwendungsfreundlichen Forschung dann vielleicht auch erbringen.

Ich danke Ihnen für das Gespräch. 